\title{
Interessante Buchempfehlungen für RadiologInnen
}

In der Mitgliederzeitschrift des BDR, DER RADIOLOGE, erscheinen jeden Monat Rezensionen von interessanten aktuellen Fachbüchern - von Mitgliedern für Mitglieder.

Aus dem Thieme-Verlag waren es zuletzt diese interessanten Werke:

\section{Arthroplasty in Hand Surgery}

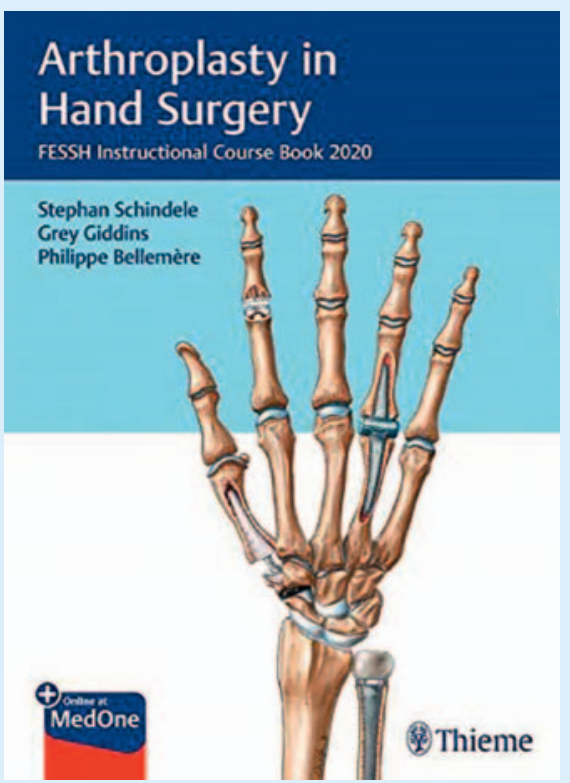

FESSH Instructional Course Book 2020, Stephan Schindele, Grey Giddins, Philippe Bellemère, 330 Seiten, Thieme, 1. Edition, 2021, Englisch, ISBN-13: 978-3132431744, $114,65 €$

Das aktuelle FESSH Instructional course book „Arthroplasty in Hand Surgery“ ist in der Reihe der regelmäßig erscheinenden Lehrbücher zu einem speziellen Schwerpunktthema unter der Schirmherrschaft der europäischen Gesellschaft für Handchirurgie FESSH (Federation of European Societies for Surgery of the Hand) erschienen.
Die Ausgabe von 2020 wurde mit Beiträgen internationaler Handchirurgen/innen mit Schwerpunkt auf den europäischen Raum aus ganz Europa von den Herausgebern Stephan Schindele (Schulthess Klinik Zürich, Schweiz), Grey Giddins (University of Bath, UK) und Pilippe Bellemère (Institute de la Main Nantes-Atlantique, Frankreich) veröffentlicht.

Die hohe Inzidenz von Arthrosen in verschiedenen Gelenken der Hand und der zunehmenden Wichtigkeit einer möglichst präzisen Handfunktion verlangt nach alternativen Behandlungsmöglichkeiten neben der ultima ratio einer Arthrodese. Nach einigen fehlgeschlagenen Implantatdesigns und Behandlungsversuchen zum Gelenkerhalt ist die dezidierte Bestandsaufnahme und Zusammenfassung möglicher Implantatversorgungen und ihrer Indikationen notwendig.

\section{Inhalt}

Das Lehrbuch ist von den Herausgebern in fünf große Abschnitte mit insgesamt 35 Kapiteln aufgeteilt.

Der erste Abschnitt beleuchtet Anatomie und Biomechanik der verschiedenen Gelenke der Hand. Herauszuheben ist hierbei das erste Kapitel - welches die beiden angesprochenen Themengebiete, die leider häufig sehr trocken und redundant abgehandelt werden, in wunderbaren Zeichnungen von Prof. Martin Langer aus Münster darstellt. Allein aufgrund dieses Kapitels ist das Buch jedem der ein Interesse an der Anatomie und Biomechanik der Fingergelenke hat, uneingeschränkt zu empfehlen. Im allgemeinen Teil folgen dann noch Grundlagen für Endoprothesendesigns, neurologischer Gelenksteuerung, subjektive und objektive Funktionsscore sowie ein Einblick in ein Prothesenregister und die Geschichte der Hand- und Fingerendoprothetik.

Der spezielle Teil gliedert sich dann in vier Abschnitte zur Endoprothetik der Fingergelenke, des Daumens, des Handgelenks sowie des distalen Radioulnargelenks. In sämtlichen Abschnitten werden die gängigen Gelenkersatzverfahren, auch biologische wie die vaskularisierte Transplantation eines Zehengelenks, beschrieben und die Indikationen bzw. Kontraindikationen aufgezeigt. Es werden aktuelle Ergebnisse aus der Literatur präsentiert sowie eigenen Erfahrungen der Autoren/innen des Kapitels, alles hochqualifizierte Handchirurgen, mit Tipps und Tricks mit dem Leser geteilt.

Sämtliche Verfahren werden mit hunderten Röntgen- und klinischen Bildern sowie immer sehr präzisen Schemazeichnungen präsentiert.

\section{Zielgruppe}

Die primäre Zielgruppe der FESSH Instructional course book Reihe sind handchirurgisch tätige Ärztinnen und Ärzte - im Falle der Endoprothetik der Finger- und Handgelenke eher fortgeschritten in der Ausbildung zur Zusatzbezeichnung Handchirurgie. Auch für den erfahrenen Handchirurgen/in bietet dieses Werk eine umfassende Übersicht zur Endoprothetik - nicht zuletzt durch den sehr lehrreichen allgemeinen Teil.

\section{Fazit}

Das Buch eignet sich aus Sicht des Autors, selbst Handchirurg, allerdings bisher ohne endoprothetische Erfahrung, für die Zielgruppe des Buches ohne Einschränkung. Für den radiologischen Fachkollegen/in ist dieses Buch aber ebenfalls zu empfehlen, falls in Patientenspektrum häufig endoprothetische Versorgungen der Finger- oder 
Handgelenke vorkommen. Zusammenfassend ist „Arthroplasty in Hand Surgery“ das aktuell wohl umfassendste und beste Werk zu diesem Themenkomplex.

Dr. med. Michael Millrose

Garmisch-Partenkirchen

\section{Top 3 Differentials in Musculoskeletal Imaging}

\section{Top 3 Differentials in} Musculoskeletal Imaging

\section{A Case Review}

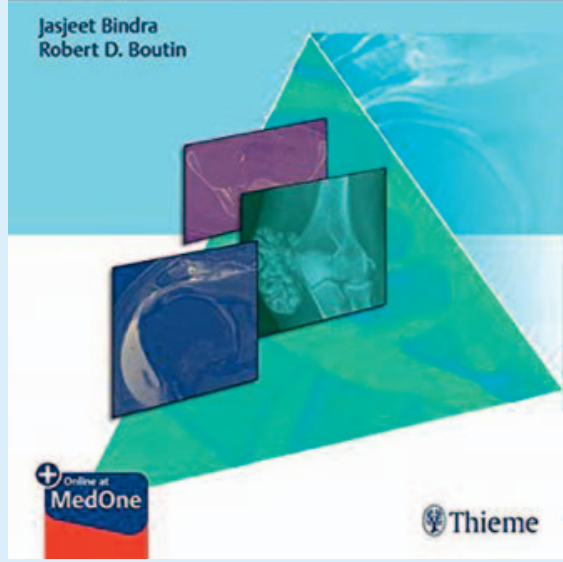

A Case Review, Jasjeet Bindra, Robert D. Boutin, 330 Seiten, Thieme Publishers New York, 1. Edition, 2021, Englisch, ISBN13: $978-1626233485,63,05 €$

Gemäß dem Motto „Häufiges ist häufig“ werden in diesem Werk über 146 Fälle präsentiert mit ihren Top 3 Differentialdiagnosen. So viel sei vorweggenommen: Ich halte diese Fallsammlung sowohl inhaltlich als auch didaktisch für durchweg gelungen und empfehle die Lektüre uneingeschränkt jedem Radiologen und jedem Arzt, der sich mit muskuloskelettaler Bildgebung beschäftigt, unabhängig von seinem Ausbildungsstand. Das Buch ist in englischer Sprache geschrieben und leicht verständlich.

Die Fälle sind in folgende 10 Themengebiete eingeteilt: Trauma, Knochentumore, obere Extremität, untere Extremität, Arthropathien, Infektionen, Weichteiltumore, Beteiligung des muskuloskelettalen Systems bei metabolischen Grunderkrankungen, Wirbelsäule und pädiatrische Erkrankungen. Das Layout des Buches folgt dem übersichtlichen Doppelseitenprinzip: Auf der linken Buchseite befinden sich die Abbildungen zu dem Fall zusammen mit den klinischen Angaben und einer Befundbeschreibung, auf der rechten Seite folgen der Schlüsselbefund „Key finding“, die Top 3 Differentialdiagnosen, die Auflösung des Falles mit der richtigen Diagnose, die „Pearls“ zur Diagnosefindung, ggf. „additional differential considerations" und eine Sammlung weiterführender Literatur.

Mit dem Erwerb des Buches erhält der Nutzer den Zugang für dieses Buch auf der online Plattform von Thieme. Dies hat den Vorteil, dass sich die Bilder bequem über den PC aufrufen und dadurch besser betrachten lassen. Die Bildselektion ist hervorragend und alle Modalitäten (Röntgen, CT und MRT) sind in Abhängigkeit vom Fall vertreten. Die Bilder selbst sind hoch qualitativ mit Geräten der neuesten Generation angefertigt, und wenn man sich die Bilder online ansieht, kann man sie vergrößern, drehen, zoomen - wie im Alltag. Toll! In der OnlineVersion sind außerdem die Literaturangaben verlinkt und teilweise kostenfrei zugänglich.

Für besonders gelungen halte ich die Aufbereitung der Top 3 Differentialdiagnosen. Kurz und fokussiert werden die relevanten klinischen, pathophysiologischen und anatomischen Grundlagen mit den wichtigsten Befunden in der Bildgebung in Zusammenhang gebracht. Der Stellenwert einzelner bildgebender Verfahren wird eingeordnet. Das dadurch entstehende Verständnis zwischen Pathomechanismus und den daraus resultierenden Veränderungen in der Bildgebung und dem klinischen Stellenwert ist etwas, das ich sowohl im Studium als auch leider in deutscher radiologischer Fachliteratur häufiger vermisst habe. Insofern haben mir die kurzen Textabschnitte über die Top 3 Differentialdiagnosen und die „Pearls“ das ein oder andere Aha-Erlebnis beschert.

\section{Fazit}

Das Buch ist durchweg gelungen und hat ein sehr gutes Preis-Leistungs-Verhältnis (69,99 Euro). Meines Erachtens nach profitieren Leser jeglichen Ausbildungsstandes von seiner Lektüre.

Dr. Katharina Schlumpberger Karlsruhe 